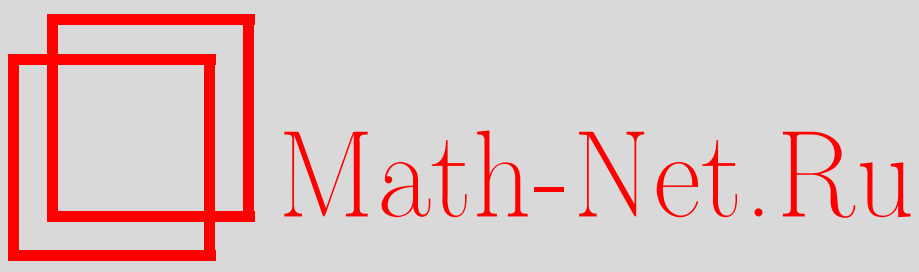

A. A. Borovkov, Integro-local theorems in boundary crossing problems for compound renewal processes, Sibirsk. Mat. Zh., 2019, Volume 60, Number 6, 1229-1246

DOI: https://doi.org/10.33048/smzh.2019.60.604

Use of the all-Russian mathematical portal Math-Net.Ru implies that you have read and agreed to these terms of use http://www . mathnet.ru/eng/agreement

Download details:

IP : 18.234 .197 .8

April 26, 2023, 09:46:46

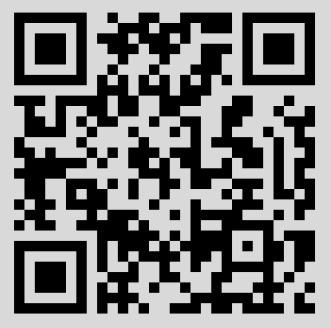


Сибирский математический журнал

Ноябрь-декабрь, 2019. Том 60, № 6

УДК 519.21

\title{
ИНТЕГРО-ЛОКАЛЬНЫЕ ТЕОРЕМЫ \\ В ГРАНИЧНЫХ ЗАДАЧАХ ДЛЯ ОБОБЩЕННЫХ ПРОЦЕССОВ ВОССТАНОВЛЕНИЯ
}

\section{А. А. Боровков}

\begin{abstract}
Аннотация. Найдена точная асимптотика вероятности того, что момент пересечения траекторией обобщенного процесса восстановления произвольной удаленной границы принадлежит заданному малому интервалу времени. В качестве важного элемента доказательства получены предельные теоремы для условного распределения скачков процесса при фиксированном конце траектории обобщенного процесса восстановления.
\end{abstract}

DOI 10.33048/smzh.2019.60.604

Ключевые слова: обобщенный процесс восстановления, большие уклонения, граничные задачи, момент пересечения удаленной границы, интегро-локальные теоремы, условное распределение скачков, задача о разорении.

\section{§ 1. Предварительные сведения}

Пусть задана последовательность независимых одинаково распределенных случайных векторов $\boldsymbol{\xi}=(\tau, \zeta), \boldsymbol{\xi}_{1}=\left(\tau_{1}, \zeta_{1}\right), \ldots$, где $\tau>0$. Обозначим

$$
T_{n}:=\sum_{j=1}^{n} \tau_{j}, \quad Z_{n}:=\sum_{j=1}^{n} \zeta_{j} \quad \text { при } \quad n \geq 1, \quad T_{0}=Z_{0}=0 .
$$

Пусть при $t \geq 0$

$$
\eta(t):=\min \left\{k \geq 0: T_{k}>t\right\}, \quad \nu(t)=\max \left\{k \geq 0: T_{k} \leq t\right\}=\eta(t)-1 .
$$

Рассмотрим обобщенные процессы восстановления (ОПВ)

$$
Z(t):=Z_{\nu(t)}, \quad Y(t):=Z_{\eta(t)}, \quad t \geq 0,
$$

и обозначим

$$
a_{\tau}=\mathbf{E} \tau, \quad a_{\zeta}=\mathbf{E} \zeta, \quad a=\frac{a_{\zeta}}{a_{\tau}}
$$

Тогда

$$
\mathbf{E} Z(t) \sim t a, \quad \mathbf{E} Y(t) \sim t a \quad \text { при } t \rightarrow \infty .
$$

Везде, если не оговорено противное, будем предполагать, что выполнено условие Крамера в следующем виде:

$[\mathbf{C}] \mathbf{E} e^{v|\boldsymbol{\xi}|}<\infty$ при некотором $v>0$.

Работа выполнена при частичной поддержке программы фундаментальных научных исследований СО РАН № I.1.3 (проект № 0314-2016-0008), а также гранта РФФИ (проект 1801-00101a).

(c) 2019 Боровков A. A. 
Кроме того, мы будем предполагать, что случайный вектор $\boldsymbol{\xi}=(\tau, \zeta)$ является нерешетчатым.

В основе результатов этой работы лежат интегро-локальные предельные теоремы для ОПВ, установленные в [1], т. е. утверждения о точной асимптотике интегро-локальных вероятностей

$$
\mathbf{P}(Z(T) \in \Delta[x))
$$

где $\Delta[x)$ есть полуинтервал $\Delta[x):=[x, x+\Delta), \Delta=\Delta_{T} \rightarrow 0$ достаточно медленно при $T \rightarrow \infty$ (сокращенно $\Delta=\bar{o}(1)$ при $T \rightarrow \infty), \alpha=x / T$ принадлежит некоторому компактному множеству (отрезку) $K$, которое будет определено ниже.

Чтобы сформулировать нужные утверждения, нам понадобится ряд предварительных сведений. Положим

$$
\mathbf{A}(\lambda, \mu)=\ln \mathbf{E} e^{\lambda \tau+\mu \zeta}, \quad \mathscr{A}=\{(\lambda, \mu): \mathbf{A}(\lambda, \mu)<\infty\}
$$

и введем в рассмотрение так называемую базовую функцию $A(\mu)$, которая определяется равенством

$$
A(\mu):=-\sup \{\lambda: A(\lambda, \mu) \leq 0\} .
$$

Если

$$
\mathbf{A}(\lambda, \mu)>0 \quad \text { при всех }(\lambda, \mu) \in \partial \mathscr{A},
$$

то $A(\mu)$ - единственное решение уравнения (относительно $\lambda$ )

$$
\mathbf{A}(-\lambda, \mu)=0,
$$

которое является аналитическим. В общем случае обозначим через $\left(\mu_{-}, \mu_{+}\right)$ максимальный интервал, содержащий точку $\mu=0$, на котором функция $A(\mu)$ аналитична. Этот интервал всегда непустой, так как $\mathbf{A}(0,0)=0$ (т. е. $A(0)=0$ ) и функция $\mathbf{A}$ аналитична в окрестности точки $(0,0)$.

Рассмотрим далее преобразование Лежандра $D(\alpha)$ над функцией $A(\mu)$ :

$$
D(\alpha)=\sup _{\mu}(\alpha \mu-A(\mu)) .
$$

Положим

$$
\alpha_{-}=A^{\prime}\left(\mu_{-}+0\right), \quad \alpha_{+}=A^{\prime}\left(\mu_{+}-0\right) .
$$

Тогда область $\left(\alpha_{-}, \alpha_{+}\right)$является областью аналитичности функции $D(\alpha)$. В широких предположениях функция $D(\alpha)$ является функцией уклонений ОПВ $Z(t)$. В правой части (1.5) sup достигается в точке $\mu(\alpha)$, которая является единственным решением уравнения

$$
A^{\prime}(\mu)=\alpha
$$

(функция $A(\mu)$ выпукла и, стало быть, $A^{\prime}(\mu)$ монотонна), так что

$$
D(\alpha)=\alpha \mu(\alpha)+\lambda(\alpha), \quad \text { где } \lambda(\alpha)=-A(\mu(\alpha)) .
$$

Свойства функций $A(\mu), D(\alpha), \mu(\alpha), \lambda(\alpha)$ детально изучены в $[1,2]$.

Чтобы определить нужный нам компакт $K$, нам понадобится еще так называемое «запретное» множество $\left(\beta_{-}, \beta_{+}\right)$. Если $\lambda_{+}=\sup \left\{\lambda: \mathbf{E} e^{\lambda \tau}<\infty\right\} \geq D(0)$, то это множество пусто. Условие $\lambda_{+} \geq D(0)$ является весьма широким, оно выполнено, например, если выполнено хотя бы одно из следующих условий (см. $[2])$. 
1. Множество $\mathscr{A}^{<0}:=\{(\lambda, \mu): \mathbf{A}(\lambda, \mu)<0\}$ вложено в полуплоскость $\lambda \leq \lambda_{+}$(это всегда так, если $\tau$ и $\zeta$ независимы).

2. $\mathbf{E} \zeta=0$.

3. Либо $\lambda_{+}=\infty$, либо функция $\mathbf{E} e^{\mu \zeta}$ всюду конечна.

Если же $\lambda_{+}<D(0)$, то, как показано в [1, лемма 2.1], функция $\lambda(\alpha)$ достигает своего максимума в точке $\alpha=0, \lambda(0)=D(0)$. Она возрастает при $\alpha<0$ и убывает при $\alpha>0, \lambda(a)=0$. Запретная область $\left[\beta_{-}, \beta_{+}\right]$определяется как максимальный отрезок, на котором $\lambda(\alpha) \geq \lambda_{+}$.

Можно показать также, что если $a>0$ и $m_{+}$- максимальное решение уравнения $A(\mu)=-\lambda_{+}$, то $\beta_{+}=A^{\prime}\left(m_{+}\right)$. Аналогичное утверждение верно при $a<0$ (см. [1]).

Появление «запретного» множества $\left[\beta_{-}, \beta_{+}\right]$связано с тем, что при $\lambda_{+}<$ $D(0)$ в области уклонений $\alpha \in\left[\beta_{-}, \beta_{+}\right]$определяющую роль начинают играть большие скачки $\tau$. Например, при $\lambda_{+}<D(0), \ln \mathbf{P}(\tau>T) \sim-\lambda_{+} T$,

$$
\ln \mathbf{P}(Z(T) \in T \Delta[0)) \geq \ln \mathbf{P}(\tau>T) \sim-T \lambda_{+} \gg-T D(0) \quad \text { при } T \rightarrow \infty,
$$

в то время как правая часть в (1.6) описывает асимптотику левой части в случае $\lambda_{+} \geq D(0)$. Вне отрезка $\left[\beta_{-}, \beta_{+}\right]$асимптотика $\ln \mathbf{P}(Z(T) \in T \Delta[\alpha))$ описывается как $-T D(\alpha)$.

Компакт $K$, который нам потребуется в приведенных ниже интегро-локальных теоремах, мы определим как отрезок

$$
K \subset\left(\alpha_{-}, \alpha_{+}\right) \backslash\left[\beta_{-}, \beta_{+}\right],
$$

содержащий окрестность точки $\alpha=a$.

Положим

$$
\begin{gathered}
\zeta(t)=\zeta_{\eta(t)}, \quad \gamma(t)=T-T_{\nu(T)}, \quad \chi(T)=T_{\eta(t)}-T, \\
\mathbf{w}=\left(w_{1}, w_{2}\right), \quad B(u, v, \mathbf{w})=\{\gamma(T) \geq u, \chi(T) \geq v, \zeta(T) \in \mathbf{w}\} .
\end{gathered}
$$

Теорема 1.1 [1]. Пусть $\boldsymbol{\xi}=(\tau, \zeta)-$ нерешетчатый случайный вектор, $\alpha=$ $x / T \in K$. Тогда при некотором $\varepsilon>0$

$$
\begin{aligned}
\mathbf{P}(Z(T) \in \Delta[x) ; B(u, v, \mathbf{w})) & \\
& =\frac{\Delta}{\sqrt{T}} C(\alpha) e^{-T D(\alpha)} I_{Z}(\alpha, u, v, \mathbf{w})(1+o(1))+O\left(e^{-T(D(\alpha)+\varepsilon)}\right),
\end{aligned}
$$

где

$$
I_{Z}(\alpha, u, v, \mathbf{w}):=\int_{u}^{\infty} e^{\lambda(\alpha) y} \mathbf{P}(\tau>y+v, \zeta \in \mathbf{w}) d y, \quad C(\alpha):=C(1, \alpha),
$$

$C(\theta, \alpha)-$ положительная непрерывная в конусе $\mathscr{D}:=\left\{(\theta, \alpha): \alpha / \theta \in\left(\alpha_{-}, \alpha_{+}\right)\right\}$ функция, определенная в соотношении (4.5) в [1], $\Delta=\Delta_{T}$ сходится к 0 достаточно медленно при $T \rightarrow \infty$, остаточные члены $о(1)$ и $O(\cdot)$ равномерны по $\alpha \in K, u \leq u_{0}$ и всем $v \geq 0$, w; здесь $u_{0}<\infty-$ произвольная постоянная.

ЗАмЕчАниЕ 1.1. В дополнение к результатам [1] нетрудно показать, что если вместо события $B(u, v, \mathbf{w})$ рассматривать в (1.7) событие $\bar{B}(u, v, \mathbf{w})=\{\gamma(T)<$ $u, \chi(T) \geq v, \zeta(T) \in \mathbf{w}\}, u \leq u_{0}$, то компакт $K$ можно расширить до любого отрезка из $\left(\alpha_{-}, \alpha_{+}\right)$(запретное множество исчезает), а множитель $I_{Z}(\alpha, u, v, \mathbf{w})$ следует заменить на $\int_{0}^{u} e^{\lambda(\alpha) y} \mathbf{P}(\tau>y+v, \zeta \in \mathbf{w}) d y$. 


\section{§ 2. Предельные теоремы для условного распределения скачков при фиксированном конце траектории}

2.1. Основная теорема. Аналогичная задача для случайных блужданий рассматривалась в $[3, \S 3.1]$. Как и в теореме 1.1 , мы будем предполагать, что нормированное уклонение $\alpha=x / T$ принадлежит компакту $K \subset\left(\alpha_{-}, \alpha_{+}\right) \backslash$ $\left[\beta_{-}, \beta_{+}\right]$, под которым будем понимать отрезок, содержащий окрестность точки $\alpha=a$. Обозначим через ${ }^{\alpha} \boldsymbol{\xi}$ преобразование Крамера над $\boldsymbol{\xi}$ в точке $(\lambda(\alpha), \mu(\alpha))$, т. е. случайный вектор с распределением

$$
\mathbf{P}\left({ }^{\alpha} \boldsymbol{\xi} \in d \boldsymbol{v}\right)=e^{\lambda(\alpha) v_{(1)}+\mu(\alpha) v_{(2)}} \mathbf{P}(\boldsymbol{\xi} \in d \boldsymbol{v}), \quad \boldsymbol{v}=\left(v_{(1)}, v_{(2)}\right),
$$

так что существует плотность ${ }^{\alpha} p(\boldsymbol{v})$ распределения ${ }^{\alpha} \boldsymbol{\xi}$ относительно распределения $\boldsymbol{\xi}$ :

$$
{ }^{\alpha} p(\boldsymbol{v}):=\frac{\mathbf{P}\left({ }^{\alpha} \boldsymbol{\xi} \in d \boldsymbol{v}\right)}{\mathbf{P}(\boldsymbol{\xi} \in d \boldsymbol{v})}=e^{\lambda(\alpha) v_{(1)}+\mu(\alpha) v_{(2)}}
$$

(напомним, что $\mathbf{A}(\lambda(\alpha), \mu(\alpha))=0)$. При произвольном фиксированном целом $m \geq 1$ обозначим через $K_{\boldsymbol{\xi}}$ произвольный компакт из $\mathbb{R}^{2 m}$. Пусть далее $\overrightarrow{\boldsymbol{v}}=$ $\left(\boldsymbol{v}_{1}, \ldots, \boldsymbol{v}_{m}\right), \boldsymbol{v}_{i} \in \mathbb{R}^{2}$ и $1 \leq k_{1} \leq \cdots<k_{m}$ - произвольный набор индексов.

Теорема 2.1. Пусть $\zeta-$ нерешетчатая случайная величина и выполнено условие $[\mathbf{C}], \alpha=x / T$. Тогда плотность

$$
p_{T, x}(\overrightarrow{\boldsymbol{v}}):=\frac{\mathbf{P}(\overrightarrow{\boldsymbol{\xi}} \in d \overrightarrow{\boldsymbol{v}} \mid Z(T) \in \Delta[x))}{\mathbf{P}\left({ }^{\alpha} \overrightarrow{\boldsymbol{\xi}} \in d \overrightarrow{\boldsymbol{v}}\right)}
$$

условного распределения $\overrightarrow{\boldsymbol{\xi}}=\left(\boldsymbol{\xi}_{k_{1}}, \ldots, \boldsymbol{\xi}_{k_{m}}\right)$ при условии $\{Z(T) \in \Delta[x)\}$ относительно распределения ${ }^{\alpha} \overrightarrow{\boldsymbol{\xi}}=\left({ }^{\alpha} \boldsymbol{\xi}_{1}, \ldots,{ }^{\alpha} \boldsymbol{\xi}_{m}\right)$, где ${ }^{\alpha} \boldsymbol{\xi}_{j}-$ независимые копии ${ }^{\alpha} \boldsymbol{\xi}$, сходится при $T \rightarrow \infty$ к 1 равномерно по $\alpha \in K, \overrightarrow{\boldsymbol{v}} \in K_{\boldsymbol{\xi}} \subset \mathbb{R}^{2 m}, \Delta \in\left[\Delta_{T}, \Delta_{0}\right]$. Здесь $\Delta_{T}=\bar{o}(1)$ при $T \rightarrow \infty\left(\right.$ т. е. $\Delta_{T} \rightarrow 0$ достаточно медленно при $\left.T \rightarrow \infty\right)$, $\Delta_{0}>0$ - любое фиксированное число.

Такое же утверждение справедливо и для набора (для вектора $\overrightarrow{\boldsymbol{\xi}}$ ) случайных индексов $\nu(T)-k_{1}, \ldots, \nu(T)-k_{m}$ при $k_{1} \geq 0$.

Из доказательства теоремы будет видно, что можно брать конечные наборы и других индексов, расположенных между 1 и $\nu(T)$.

Теорема 2.1 означает сходимость по вариации условных распределений $\overrightarrow{\boldsymbol{\xi}}$ при условии $\{Z(T) \in \Delta[x)\}$ к распределению ${ }^{\alpha} \overrightarrow{\boldsymbol{\xi}}$. В частности, из нее вытекает

Следствие 2.1. Пусть выполнены условия теоремы 2.1. Тогда для любых борелевских множеств $B_{1}, \ldots, B_{m}$ из $\mathbb{R}^{2}$

$$
\mathbf{P}\left(\boldsymbol{\xi}_{1} \in B_{1}, \ldots, \boldsymbol{\xi}_{m} \in B_{m} \mid Z(T) \in \Delta[x)\right)=\prod_{i=1}^{m} \mathbf{P}\left({ }^{\alpha} \boldsymbol{\xi}_{i} \in B_{i}\right)+\varepsilon_{T},
$$

где $\varepsilon_{T} \rightarrow 0$ при $T \rightarrow \infty$ равномерно по $\alpha \in K, \Delta \in\left[\Delta_{T}, \Delta_{0}\right]$.

В дальнейшем главную часть в правой части (2.3) мы будем называть «предельным распределением» при $T \rightarrow \infty$, хотя это не совсем корректно, так как значение $\alpha \in K$ может зависеть от $T$. Такого же соглашения мы будем придерживаться и в ряде других аналогичных случаях. Формально термин «предельное распределение» в (2.3) оправдан лишь в тех случаях, когда $\alpha \rightarrow \alpha_{0} \in K$ при $T \rightarrow \infty$ и $\alpha$ в правой части (2.3) заменено на $\alpha_{0}$. Более корректно было бы 
в соотношениях (2.3) говорить о «близких распределениях». Эта более точная терминология была введена и использовалась в $\S 3.3$ в [4] при изучении аналогичных задач для случайных блужданий. Однако мы, чтобы сделать изложение более кратким, ниже будем следовать (не совсем корректно) названному выше соглашению о понимании слов «предельное распределение».

ДОКАЗАТЕЛЬСТВО ТЕОРЕМЫ 2.1. ОбозНачим

$$
\sum_{i=1}^{m} \boldsymbol{v}_{i}=(t, z)
$$

Тогда при $t \leq t_{0}<T$

$$
\begin{array}{r}
p_{T, x}^{0}(\overrightarrow{\boldsymbol{v}}):=\frac{\mathbf{P}(\overrightarrow{\boldsymbol{\xi}} \in d \overrightarrow{\boldsymbol{v}} \mid Z(T) \in \Delta[x))}{\mathbf{P}(\overrightarrow{\boldsymbol{\xi}} \in d \overrightarrow{\boldsymbol{v}})}=\frac{\mathbf{P}(\overrightarrow{\boldsymbol{\xi}} \in d \overrightarrow{\boldsymbol{v}}, Z(T) \in \Delta[x))}{\mathbf{P}(\overrightarrow{\boldsymbol{\xi}} \in d \overrightarrow{\boldsymbol{v}}) \mathbf{P}(Z(T) \in \Delta[x))} \\
=\frac{\mathbf{P}(Z(T-t) \in \Delta[x-z))}{\mathbf{P}(Z(T) \in \Delta[x))} .
\end{array}
$$

В силу теоремы 1.1

$$
\mathbf{P}(Z(T) \in \Delta[x))=\frac{\Delta}{\sqrt{T}} C(\alpha) e^{-T D(\alpha)} I_{Z}(\alpha, 0,0, \mathbb{R})(1+o(1)),
$$

где $\alpha=x / T, C(\alpha)$ - положительная непрерывная функция на $K$,

$$
I_{Z}(\alpha, 0,0, \mathbb{R})=\int_{0}^{\infty} e^{\lambda(\alpha) y} \mathbf{P}(\tau>y) d y,
$$

$\Delta=\Delta_{T}=\bar{o}(1)$ при $T \rightarrow \infty$, остаточный член $o(1)$ равномерен по $\alpha \in K$.

Положим

$$
\alpha_{m}:=\frac{x-z}{T-t}=\alpha-\frac{z}{T}+\frac{\alpha t}{T}+O\left(T^{-2}\right) .
$$

Так как $\alpha_{m}=\alpha+o(1)$ при $T \rightarrow \infty$ и функция $C(\alpha)$ в теореме 1.1 есть положительная непрерывная функция от $\alpha$, то, применяя эту теорему к числителю и знаменателю в (2.4), получим

$$
p_{T, x}^{0}(\overrightarrow{\boldsymbol{v}})=\frac{I_{Z}\left(\alpha_{m}, 0,0, \mathbb{R}\right)}{I_{Z}(\alpha, 0,0, \mathbb{R})} \exp \left\{-(T-t) D\left(\alpha_{m}\right)+T D(\alpha)\right\}(1+o(1)) .
$$

Поскольку $\boldsymbol{v} \in K_{\boldsymbol{\xi}}$, то $t \leq t_{0},|z| \leq z_{0}$ при некоторых $t_{0}, z_{0}$ и

$$
D\left(\alpha_{m}\right)=D\left(\alpha-\frac{z}{T}+\frac{\alpha t}{T}+O\left(T^{-2}\right)\right)=D(\alpha)+\mu(\alpha)\left(\frac{\alpha t}{T}-\frac{z}{T}\right)+O\left(T^{-2}\right),
$$

где остаточный член равномерен по $\boldsymbol{v} \in K_{\boldsymbol{\xi}}$ при $T \rightarrow \infty$. Поэтому

$$
p_{T, x}^{0}(\overrightarrow{\boldsymbol{v}})=\exp \{t D(\alpha)-\mu(\alpha)(\alpha t-z)\}(1+o(1)) .
$$

Так как $D(\alpha)=\alpha \mu(\alpha)-A(\mu(\alpha))$, то в (2.7) выражение в фигурных скобках равно

$$
z \mu(\alpha)+t(D(\alpha)-\alpha \mu(\alpha))=z \mu(\alpha)-t A(\mu(\alpha))=z \mu(\alpha)+t \lambda(\alpha) .
$$

Кроме того,

$$
\frac{I_{Z}\left(\alpha_{m}, 0,0, \mathbb{R}\right)}{I_{Z}(\alpha, 0,0, \mathbb{R})} \rightarrow 1 \quad \text { при } T \rightarrow \infty
$$


Поэтому в силу $(2.1),(2.8)$

$$
\begin{aligned}
p_{T, x}^{0}(\overrightarrow{\boldsymbol{v}})= & \prod_{i=1}^{m} \frac{\mathbf{P}\left({ }^{\alpha} \boldsymbol{\xi}_{i} \in d \boldsymbol{v}_{i}\right)}{\mathbf{P}\left(\boldsymbol{\xi}_{i} \in d \boldsymbol{v}_{i}\right)}(1+o(1))=\frac{\mathbf{P}\left({ }^{\alpha} \overrightarrow{\boldsymbol{\xi}} \in d \overrightarrow{\boldsymbol{v}}\right)}{\mathbf{P}(\overrightarrow{\boldsymbol{\xi}} \in d \overrightarrow{\boldsymbol{v}})}(1+o(1)), \\
& p_{T, x}(\overrightarrow{\boldsymbol{v}})=p_{T, x}^{0}(\overrightarrow{\boldsymbol{v}}) \frac{\mathbf{P}(\overrightarrow{\boldsymbol{\xi}} \in d \overrightarrow{\boldsymbol{v}})}{\mathbf{P}\left({ }^{\alpha} \overrightarrow{\boldsymbol{\xi}} \in d \overrightarrow{\boldsymbol{v}}\right)}=1+o(1),
\end{aligned}
$$

где остаточный член равномерен по $\alpha \in K, \overrightarrow{\boldsymbol{v}} \in K_{\boldsymbol{\xi}}$.

Доказательство второго утверждения теоремы повторяет приведенные выше рассуждения. Теорема 2.1 доказана.

ЗАмЕЧАнИЕ 2.1. В соответствии с теоремой 1.1 предельное условное распределение $\overrightarrow{\boldsymbol{\xi}}$ при условии $\{Z(T) \in \Delta[x), B(u, v, \mathbf{w})\}$ останется таким же, как в теореме 2.1. Действительно, вместо множителя $I_{Z}(\alpha, 0,0, \mathbb{R})$ в $(2.5)$ теперь появится множитель $I_{Z}(\alpha, u, v, \mathbf{w})$. Если он не равен нулю, то отношение множителей этого вида

$$
\frac{I_{Z}\left(\alpha_{m}, u, v, \mathbf{w}\right)}{I_{Z}(\alpha, u, v, \mathbf{w})}
$$

(это аналог соотношения (2.6)) сходится, как и в (2.8), к 1 при $T \rightarrow \infty$ и все проведенные в доказательстве теоремы 2.1 выкладки сохранятся.

Очевидно, что «предельное» условное распределение $\overrightarrow{\boldsymbol{\xi}}$ будет таким же и в том случае, если событие $B(u, v, \mathbf{w})$ (см. теорему 1.1) заменить событием о принадлежности вектора $(\gamma(T), \chi(T), \zeta(T))$ произвольному параллелепипеду положительной меры, порожденной распределением

$$
\frac{I_{Z}(\alpha, u, v, \mathbf{w})}{I_{Z}(\alpha, 0,0, \mathbb{R})} .
$$

Если параллелепипед ограничен по $u$, то в соответствии с замечанием 1.1 в качестве компакта $K$ в теореме 2.1 можно брать любой отрезок из $\left(\alpha_{-}, \alpha_{+}\right)$.

Все изложенное, конечно же, распространяется и на векторы $\vec{\xi}$ со случайными индексами, названными во втором утверждении теоремы 2.1.

Утверждение, аналогичное теореме 2.1 , можно установить и для распределения $\overrightarrow{\boldsymbol{\xi}}$ при условии $\{Y(T) \in \Delta[x)\}$. Но основной интерес в приложениях представляют процессы $Z(t)$. Кроме того, формулировки утверждений, аналогичных теореме 2.1, и других для ОПВ $Y(t)$ выглядят более сложно (см. [1]). Поэтому в дальнейшем мы ограничимся рассмотрением главным образом процессов $Z(t)$.

2.2. О распределении вектора ${ }^{\alpha} \boldsymbol{\xi}$. Преобразование Лапласа над распределением ${ }^{\alpha} \boldsymbol{\xi}$ имеет вид

$$
{ }^{\alpha} \mathbf{A}(\lambda, \mu):=\ln \mathbf{E} e^{\left\langle{ }^{\alpha} \boldsymbol{\xi},(\lambda, \mu)\right\rangle}=\mathbf{A}(\lambda+\lambda(\alpha), \mu+\mu(\alpha)) .
$$

Базовая функция ${ }^{\alpha} A(\mu)$, соответствующая скачкам ${ }^{\alpha} \boldsymbol{\xi}$ в окрестности точки $(0,0)$, есть решение (со знаком минус) уравнения $\mathbf{A}(\lambda+\lambda(\alpha), \mu+\mu(\alpha))=0$, т. e.

$$
{ }^{\alpha} A(\mu)=\lambda(\alpha)+A(\mu+\mu(\alpha)),
$$

так что ${ }^{\alpha} A(0)=0$. Среднее значение процесса ${ }^{\alpha} Z(t)$ со скачками ${ }^{\alpha} \boldsymbol{\xi}$ на единицу времени асимптотически эквивалентно значению

$$
{ }^{\alpha} a:={ }^{\alpha} A^{\prime}(0)=A^{\prime}(\mu(\alpha))=\alpha
$$

(напомним, что $\mu(\alpha)$ есть функция, обратная к $A^{\prime}(\mu)$ ). Дисперсия ${ }^{\alpha} Z(t)$ на единицу времени асимптотически эквивалентна

$$
{ }^{\alpha} \sigma^{2}=A^{\prime \prime}(\mu(\alpha)) \text {. }
$$




\section{$\S 3$. Интегро-локальные теоремы для времени первого прохождения траекторией ОПВ высокого уровня}

Обозначим

$$
\eta_{Z}(x):=\min \{t: Z(t)>x\}, \quad \chi_{Z}(x)=Z\left(\eta_{Z}(x)\right)-x .
$$

Предметом изучения в этом разделе будет асимптотика вероятности

$$
\mathbf{P}\left(\eta_{Z}(x) \in \delta[T), \chi_{Z}(x) \in \Delta[v)\right) \quad \text { при } \alpha=\frac{x}{T}>a+\varepsilon
$$

и некотором $\varepsilon>0$. Чтобы сформулировать основное утверждение, введем в рассмотрение ОПВ ${ }^{\alpha} Y(t)$, как ОПВ $Y(t)$, определенный в $\S 1$, но со скачками $\left({ }^{\alpha} \tau,-{ }^{\alpha} \zeta\right)$, где, как и прежде, $\left({ }^{\alpha} \tau,{ }^{\alpha} \zeta\right)={ }^{\alpha} \boldsymbol{\xi}$ :

$$
{ }^{\alpha} Y(t)=-{ }^{\alpha} Z_{\eta(t)}, \quad{ }^{\alpha} Z_{n}=\sum_{j=1}^{n}{ }^{\alpha} \zeta_{j},
$$

${ }^{\alpha} \zeta_{j}-$ независимые копии ${ }^{\alpha} \zeta$. В силу (2.9)

$$
\mathbf{E}^{\alpha} Y(t) \sim-{ }^{\alpha} a t=-\alpha t \quad \text { при } t \rightarrow \infty .
$$

Поэтому для ${ }^{\alpha} \bar{Y}=\sup _{t \geq 0}{ }^{\alpha} Y(t)$ при $\alpha \geq \alpha_{0}>0$ имеем

$$
\mathbf{P}\left({ }^{\alpha} \bar{Y} \leq-v\right)>0
$$

при $v>0$ таких, что $\mathbf{P}\left(\zeta_{1}<-v\right)>0$ (напомним, что $\left.{ }^{\alpha} Y(0)=-{ }^{\alpha} \zeta_{1}\right)$.

Теорема 3.1. Пусть $\boldsymbol{\xi}=(\tau, \zeta)$ - нерешетчатый вектор, $\alpha=x / T>a+\varepsilon$ при некотором $\varepsilon>0$. Тогда

$$
\begin{aligned}
\mathbf{P}\left(\eta_{Z}(x) \in \delta[T), \chi_{Z}(x)\right. & \in \Delta[v)) \\
=\mathbf{P}(Z(T+\delta) \in & \Delta[x+v), \gamma(T+\delta)<\delta) \mathbf{P}\left({ }^{\alpha} \bar{Y} \leq-v\right)(1+o(1)) \\
& =\frac{\Delta \delta C(\alpha)}{\sqrt{T}} e^{-T D(\alpha)-v \mu(\alpha)} \mathbf{P}\left({ }^{\alpha} \bar{Y} \leq-v\right)(1+o(1)),
\end{aligned}
$$

где $C(\alpha)$ - положительная непрерывная функция, определенная в теореме 1.1, $\Delta=\Delta_{T}=\bar{o}(1), \delta=\delta_{T}=\bar{o}(1)$ при $T \rightarrow \infty$, остаточный член о(1) равномерен по $\alpha \in K \cap[\varepsilon, \infty), \varepsilon>0$; компакт $K$ определен перед теоремой 1.1.

В соответствии с замечанием 1.1 здесь в качестве компакта $K$ можно брать любой отрезок из $\left(\alpha_{-}, \alpha_{+}\right)$, содержащий точку $a$.

ДокАЗАТЕльСтво. Положим

$$
B_{Z, T}=\left\{\eta_{Z}(x) \in \delta[T), \chi_{Z}(x) \in \Delta[v)\right\} .
$$

ОценкА $\mathbf{P}\left(B_{Z, T}\right)$ снизу. Положим $\bar{Z}(T)=\max _{0 \leq t \leq T} Z(t)$. Тогда

$B_{Z, T, \gamma}:=\{\bar{Z}(T+\delta-\gamma(T+\delta)-0) \leq x, Z(T+\delta) \in x+\Delta[v), \gamma(T+\delta)<\delta\} \subset B_{Z, T}$.

Поэтому

$$
\mathbf{P}\left(B_{Z, T}\right) \geq \mathbf{P}\left(B_{Z, T, \gamma}\right)
$$

ОцЕНКА СВЕРХУ. Имеем

$$
B_{Z, T}=\{Y(T) \in x+\Delta(v), \chi(T)<\delta, \bar{Z}(T+\chi(T)-0) \leq x\}
$$


т. е. событие $B_{Z, T}$ измеримо относительно $\sigma$-алгебры, порожденной процессом $Y(T)$ до марковского момента $\eta(T)$ включительно, так что события $B_{Z, T}$ и случайная величина $\tau_{\eta(T)+1}$ независимы. С другой стороны, в силу (3.4)

$$
\begin{aligned}
& B_{Z, T} \cap\left\{\tau_{\eta(T)+1} \geq \delta\right\} \\
& \subset\{Z(T+\delta) \in x+\Delta[v), \gamma(T+\delta)<\delta, \bar{Z}(T+\delta-\chi(T+\delta)-0) \leq x\}=B_{Z, T, \gamma} \\
& \quad \mathbf{P}\left(B_{Z, T}\right) \mathbf{P}(\tau \geq \delta) \leq \mathbf{P}\left(B_{Z, T, \gamma}\right) .
\end{aligned}
$$

Так как в дальнейшем в качестве $\delta$ мы будем рассматривать последовательность $\delta=\delta_{T} \rightarrow 0$ (достаточно медленно при $T \rightarrow \infty$ ), то $\mathbf{P}(\tau \geq \delta)=1+o(1)$ при $T \rightarrow \infty$ и в силу $(3.3),(3.5)$

$$
\mathbf{P}\left(B_{Z, T}\right)=\mathbf{P}\left(B_{Z, T, \gamma}\right)(1+o(1)) \quad \text { при } T \rightarrow \infty .
$$

Далее имеем

$$
\begin{aligned}
& \mathbf{P}\left(B_{Z, T, \gamma}\right)=\mathbf{P}(Z(T+\delta) \in \Delta[x+v), \gamma(T+\delta)<\delta) \\
& \quad \times \mathbf{P}(\bar{Z}(T+\delta-\gamma(T+\delta)-0) \leq x \mid Z(T+\delta) \in \Delta[x+v), \gamma(T+\delta)<\delta) .
\end{aligned}
$$

Так как

$$
I_{Z}(\alpha, 0,0, \mathbb{R})-I_{Z}(\alpha, \delta, 0, \mathbb{R})=\int_{0}^{\delta} e^{\lambda(\alpha) y} \mathbf{P}(\tau>y) d y \sim \delta
$$

при $\delta \rightarrow 0$ и

$$
T D\left(\frac{x+v}{T}\right)=T D(\alpha)+\mu(\alpha) v+o(1)
$$

при $T \rightarrow \infty$, то асимптотика первого сомножителя в правой части (3.7) в силу теоремы 1.1 совпадает с асимптотикой произведения

$$
\frac{\Delta \delta C(\alpha)}{\sqrt{T}} e^{-T D(\alpha)-v \mu(\alpha)}
$$

(cp. c (3.1)).

Покажем теперь, что второй сомножитель в правой части (3.7) асимптотически эквивалентен $\mathbf{P}\left({ }^{\alpha} \bar{Y} \leq-v\right)$. При этом наше изложение доказательства будет отчасти схематичным, поскольку (а) оно весьма прозрачно и следует той же схеме, которая использовалась в $[3, \S 3.3]$ при доказательстве аналогичного факта для случайных блужданий, (b) переход к более детальному изложению существенных трудностей не вызывает.

В силу теоремы 2.1 и замечания 2.1 условное распределение скачков $\boldsymbol{\xi}_{\nu(T+\delta)}$, $\boldsymbol{\xi}_{\nu(T+\delta)-1}, \ldots, \boldsymbol{\xi}_{\nu(T+\delta)-m-1}$ при условии $\{Z(T+\delta) \in \Delta[x+v), \gamma(T+\delta)<\delta\}$ будет иметь «предельное» условное распределение, совпадающее с распределением $\left({ }^{\alpha} \boldsymbol{\xi}_{1}, \ldots,{ }^{\alpha} \boldsymbol{\xi}_{m}\right)$, где ${ }^{\alpha} \boldsymbol{\xi}_{j}$ независимы. Если рассматривать процесс $Z(t)$ в обратном направлении от точки $T+\delta-\gamma(T+\delta)$, т. е. процесс $Z(T+\delta-\gamma(T+\delta)-u)$, $u>0$, то на событии $\left\{{ }^{\alpha} \bar{Y} \leq-v\right\}$ он будет начинаться с отрицательного скачка $-{ }^{\alpha} \zeta_{1} \leq-v$. На первых $m$ «шагах» предельный процесс в обратном направлении ${ }^{\alpha} Y(u)$ будет иметь вид процесса $Y(u)$ со скачками $\left({ }^{\alpha} \tau_{1},-{ }^{\alpha} \zeta_{1}\right), \ldots,\left({ }^{\alpha} \tau_{m},-{ }^{\alpha} \zeta_{m}\right)$. Выбором большого $m$ вероятность $\mathbf{P}\left({ }^{\alpha} \bar{Z}_{m} \leq-v\right)$, где ${ }^{\alpha} \bar{Z}_{m}=\max _{k \leq m} \sum_{j=1}^{k}\left(-{ }^{\alpha} \zeta_{j}\right)$, может быть сделана сколь угодно близкой к $\mathbf{P}\left({ }^{\alpha} \bar{Y} \leq-v\right),{ }^{\alpha} \bar{Y}=\sup _{u \geq 0}{ }^{\alpha} Y(u)$. Другими словами, выбором $N$ вероятность $\mathbf{P}\left({ }^{\alpha} \bar{Y}(N) \leq-v\right)$, где ${ }^{\alpha} \bar{Y}(N)=\max _{u \leq N}{ }^{\alpha} Y(u)$, может быть сделана сколь угодно близкой к $\mathbf{P}\left({ }^{\alpha} \bar{Y} \leq-v\right)$. 
Остается установить, что условная вероятность пересечь процессу $Z(t)$ границу $x$ на интервале времени $(0, T+\delta-M)$ при условии $\{Z(T+\delta) \in \Delta[x+$ $v), \gamma(T+\delta)<\delta\}$ пренебрежимо мала при больших $M$. Точнее, нам достаточно установить пренебрежимую малость при больших $T, M$ и $T-M$ условной вероятности

$$
\begin{aligned}
P^{\text {cond }}:=\mathbf{P}\left(\eta_{Z}(x)<T\right. & -M \mid Z(T) \in \Delta[x+v), \gamma(T)<\delta) \\
& =\frac{\mathbf{P}\left(\eta_{Z}(x)<T-M, Z(T) \in \Delta[x+v), \gamma(T)<\delta\right)}{\mathbf{P}(Z(T) \in \Delta[x+v), \gamma(T)<\delta)} .
\end{aligned}
$$

Положим $t_{k}=k h, k=0,1, \ldots, N ; h[t):=[t, t+h)$, считая $N=\frac{T-M}{h}$ целым числом. Так как $\eta_{Z}(x)$ - марковский момент, числитель в правой части (3.8) равен

$$
\sum_{k=0}^{N} \int_{t_{k}}^{t_{k+1}} \mathbf{P}\left(\eta_{Z}(x) \in d t\right) \mathbf{P}\left(Z^{*}(T-t) \in \Delta[x+v), \gamma(T-t)<\delta\right),
$$

где процесс $Z^{*}$ устроен так же, как $Z$, и не зависит от $Z$. В силу теоремы 1.1 второй множитель в (3.9) можно заменить на

$$
\mathbf{P}\left(Z\left(T-t_{k}\right) \in \Delta[x+v), \gamma\left(T-t_{k}\right)<\delta\right)\left(1+\varepsilon_{h}\right),
$$

где $\varepsilon_{h} \rightarrow 0$ при $h \rightarrow 0$, так что полученное выражение отличается от $\mathbf{P}(Z(T-$ $\left.\left.t_{k}\right) \in \Delta[x+v)\right)$ множителем $\delta(1+o(1))$ при малых $\delta$. Последнее высказывание относится и к знаменателю в (3.8). В итоге получаем, что при малых $h, \delta$

$$
P^{\text {cond }}=\sum_{k=0}^{N} \frac{\mathbf{P}\left(\eta_{Z}(x) \in h\left[t_{k}\right)\right) \mathbf{P}\left(Z\left(T-t_{k}\right) \in \Delta[x+v)\right)(1+o(1))}{\mathbf{P}(Z(T) \in \Delta[x+v))} .
$$

Числители в $(3.8),(3.10)$ означают вероятность пучка «искривленных» траекторий (проходящих выше точки $(T-M, x)$ ), соединяющих точку $(0,0)$ с окрестностью точки $(T, x+v)$. Знаменатель в этих соотношениях равен вероятности всех траекторий, соединяющих названные области, в том числе «прямолинейные», наиболее вероятные траектории. Это означает, что числитель в (3.8), (3.10) в силу строгой выпуклости функции $D$ экспоненциально (по $M$ ) меньше знаменателя, так что вероятность $P^{c o n d}$ мала при больших $M$. Чтобы получить явную оценку для $P^{\text {cond }}$, заметим, что так как $\eta_{Z}(x)-$ марковский момент, то следующий за ним горизонтальный скачок $\tau^{*}$ не зависит от предыстории и распределен как $\tau$. Поэтому

$$
\mathbf{P}\left(\eta_{Z}(x) \in h\left[t_{k}\right), \tau^{*}>h\right)=\mathbf{P}\left(\eta_{Z}(x) \in h\left[t_{k}\right)\right) \mathbf{P}(\tau>h) .
$$

С другой стороны,

$$
\left\{\eta_{Z}(x) \in h\left[t_{k}\right), \tau^{*}>h\right\} \subset\left\{Z\left(t_{k+1}\right)>x\right\}
$$

и, стало быть, для $h$ таких, что $\mathbf{P}(\tau>h)>1 / 2$, получаем

$$
\mathbf{P}\left(\eta_{Z}(x) \in h\left[t_{k}\right)\right) \leq \frac{\mathbf{P}\left(Z\left(t_{k+1}\right)>x\right)}{\mathbf{P}(\tau>h)} \leq 2 \mathbf{P}\left(Z\left(t_{k+1}\right)>x\right) .
$$

Поэтому в силу (3.10)

$$
P^{\text {cond }} \leq \sum_{k=0}^{N} \frac{2 \mathbf{P}\left(Z\left(t_{k+1}\right)>x\right) \mathbf{P}\left(Z\left(T-t_{k}\right) \in \Delta[x+v)\right)}{\mathbf{P}(Z(T) \in \Delta[x+v))}(1+o(1)) .
$$


Остается воспользоваться асимптотическими представлениями для всех элементов правой части в (3.11), которые можно извлечь из теоремы 1.1. Мы предоставляем это читателю. Теорема 3.1 доказана.

Обозначим

$$
P_{\alpha}(v)=\mathbf{P}\left({ }^{\alpha} \bar{Y}<v\right), \quad J_{\alpha}(v)=\int_{0}^{v} e^{-v \mu(\alpha)} P_{\alpha}(-u) d u .
$$

Положим в теореме $3.1 v=v_{k}=k \Delta, k=0,1, \ldots$, и просуммируем полученные соотношения по $k$ от 0 до $N=v / \Delta$ (считаем $N$ целым). В левой части получим вероятность $\mathbf{P}\left(\eta_{Z}(x) \in \delta[T), \chi_{Z}(x)<v\right)$, а в правой - значение

$$
\frac{\delta C(\alpha)}{\sqrt{T}} e^{-T D(\alpha)} \sum_{k=0}^{N} \Delta e^{-v_{k} \mu(\alpha)} P_{\alpha}\left(-v_{k}\right)(1+o(1)),
$$

где интегральная сумма соответствует интервалу $J_{\alpha}(v)$. Мы получили

Следствие 3.1. Пусть выполнены условия теоремы 3.1. Тогда

$$
\mathbf{P}\left(\eta_{Z}(x) \in \delta[T), \chi_{Z}(x)<v\right)=\frac{\delta C(\alpha)}{\sqrt{T}} e^{-T D(\alpha)} J_{\alpha}(v)(1+o(1)),
$$

где $\delta=\bar{o}(1)$ при $T \rightarrow \infty$, остаточный член равномерен по $\alpha \in K \cap\{\alpha \geq \varepsilon\}, \varepsilon>0$.

В связи со следствием 3.1 представляет интерес

Лемма 3.1. Интеграл $J_{\alpha}(\infty)$ сходится при всех $\alpha \in\left(\alpha_{-}, \alpha_{+}\right) \backslash\left[\beta_{-}, \beta_{+}\right]$.

ДокАЗАТЕЛЬСтво. Заметим, что

$$
P_{\alpha}(-u)<\mathbf{P}\left({ }^{\alpha} \zeta \geq u\right)
$$

Поэтому

$$
\begin{aligned}
& J_{\alpha}(\infty)<\int_{0}^{\infty} e^{-u \mu(\alpha)} \int_{u}^{\infty} \mathbf{P}\left({ }^{\alpha} \zeta \in d t\right) d u=\int_{t=0}^{\infty} \int_{u=0}^{t} e^{-u \mu(\alpha)} d u \mathbf{P}\left({ }^{\alpha} \zeta \in d t\right) \\
& =\int_{0}^{\infty} \frac{\left(1-e^{-t \mu(\alpha)}\right)}{\mu(\alpha)} \mathbf{P}\left({ }^{\alpha} \zeta \in d t\right)=\frac{1}{\mu(\alpha)}\left(\mathbf{P}\left({ }^{\alpha} \zeta>0\right)-\mathbf{E}\left(e^{-\mu(\alpha)^{\alpha} \zeta} ;{ }^{\alpha} \zeta \geq 0\right),\right.
\end{aligned}
$$

где

$$
\mathbf{E}\left(e^{-\mu(\alpha)^{\alpha} \zeta} ;{ }^{\alpha} \zeta \geq 0\right) \leq \mathbf{E} e^{-\mu(\alpha)^{\alpha} \zeta}=\mathbf{A}(\lambda(\alpha), 0)<\infty,
$$

так как $\alpha \notin\left[\beta_{-}, \beta_{+}\right]$и, следовательно, $\lambda(\alpha)<\lambda_{+}$.

Если $\alpha$ отделено от $a$ и, стало быть, $|\mu(\alpha)|>\varepsilon$ при некотором $\varepsilon>0$, то, очевидно, $J_{\alpha}(\infty)<\infty$. Если $\alpha \rightarrow a$ при $T \rightarrow \infty$, то $\mu(\alpha) \rightarrow 0$ и

$$
J_{\alpha}(\infty) \rightarrow \mathbf{E}\left({ }^{\alpha} \zeta{ }^{\alpha} \zeta \geq 0\right)<\infty,
$$

так как ${ }^{\alpha} \zeta$ удовлетворяет условию Крамера. Лемма 3.1 доказана. 


\section{$\S 4$. Интегро-локальные теоремы в граничных задачах для обобщенных процессов восстановления}

4.1. Интегро-локальные теоремы в первой граничной задаче. Из доказательства теоремы 3.1 видно, что ее утверждение может быть обобщено на время

$$
\eta_{Z, g}=\min \left\{t: Z(t)>g_{T}(t)\right\}
$$

первого прохождения траекторией ОПВ произвольной удаленной границы $g_{T}(t)$, где $g_{T}(t)-$ функция, зависящая от растущего параметра $T$, такая, что $x:=$ $g_{T}(T) \rightarrow \infty$ при $T \rightarrow \infty$. Например, функция

$$
g_{T}(t)=T g(t / T)
$$

где $g(u)$ - фиксированная функция такая, что $g(1)>0$, обладает этим свойством.

Не ограничивая общности, будем изучать асимптотику того, что пересечение границы $g_{T}$ произойдет в окрестности точки $t=T$, при этом будем предполагать, что функции $g_{T}(t)$ удовлетворяют следующим условиям.

(a) Функция $g_{T}(t)$ асимптотически линейна в окрестности точки $t=T$, т. е. для любого фиксированного $N>0$, некоторого $g^{\prime}<\alpha$ и $T \rightarrow \infty$ выполняется

$$
g_{T}(t)=x+(t-T) g^{\prime}+o(1) \quad \text { при }|t-T| \leq N, \alpha=\frac{x}{T} \in K .
$$

Если, например, функция $g_{T}(t)$ имеет вид (4.2) и дифференцируема в точке $t=1$, то $g^{\prime}=g^{\prime}(1)$.

(b) В области $t \leq T-N$ при некотором $r^{\prime}<g^{\prime}$ выполняется

$$
g_{T}(t) \geq x+r^{\prime}(t-T)>0 .
$$

Отметим, что если в этих условиях $g^{\prime}<0$, то возникает качественно новая возможность, состоящая в том, что процесс $Z(t)$ может пересечь границу $g_{T}(t)$ не только вертикальным, но и горизонтальным скачком.

Обозначим через $A^{\uparrow}\left(A^{\rightarrow}\right)$ событие, состоящее в том, что пересечение границы $g_{T}(t)$ процессом $Z(T)$ произошло вертикальным (горизонтальным) скачком. Мы найдем асимптотику вероятностей

$$
\mathbf{P}\left(\eta_{Z, g} \in \Delta[T), A^{\uparrow}\right) \quad \text { и } \quad \mathbf{P}\left(\eta_{Z, g} \in \Delta[T), A^{\rightarrow}\right)
$$

и тем самым асимптотику вероятностей

$$
\mathbf{P}\left(\eta_{Z, g} \in \Delta[T)\right)=\mathbf{P}\left(\eta_{Z, g} \in \Delta[T), A^{\uparrow}\right)+\mathbf{P}\left(\eta_{Z, g} \in \Delta[T), A^{\rightarrow}\right)
$$

при $T \rightarrow \infty$. На самом деле будут изучены вероятности более информативных событий, чем в (4.5).

1. Начнем с вероятностей пересечения границы вертикальным скачком.

Положим

$$
\chi_{Z, g}:=Z\left(\eta_{Z, g}\right)-g_{T}\left(\eta_{Z, g}\right) .
$$

Теорема 4.1. Пусть $\boldsymbol{\xi}-$ нерешетчатый вектор, $\alpha=\frac{x}{T}>a+\varepsilon$ и выполнены условия (a), (b) при некоторых $g^{\prime}<\alpha-\varepsilon$ и $\varepsilon>0$. Тогда

$$
\begin{array}{r}
\mathbf{P}\left(\eta_{Z, g} \in \delta[T), A^{\uparrow}, \chi_{Z, g} \in \Delta[v)\right) \\
=\mathbf{P}(Z(T+\delta) \in \Delta[x+v), \gamma(T+\delta)<\delta) P_{\alpha, g^{\prime}}(-v)(1+o(1)) \\
=\frac{\Delta \delta C(\alpha)}{\sqrt{T}} e^{-T D(\alpha)-v \mu(\alpha)} P_{\alpha, g^{\prime}}(-v)(1+o(1)),
\end{array}
$$


где

$$
P_{\alpha, g^{\prime}}(-v):=\mathbf{P}\left(\sup _{t \geq 0}\left({ }^{\alpha} Y(t)+g^{\prime} t\right) \leq-v\right),
$$

$\Delta=\Delta_{T}=\bar{o}(1), \delta=\delta_{T}=\bar{o}(1)$ при $T \rightarrow \infty$, остаточный член $о(1)$ равномерен по $\alpha \in K \cap[\varepsilon, \infty), \varepsilon>0$; компакт $K$ определен перед теоремой 1.1 .

Если $g^{\prime} \geq 0$, то $\mathbf{P}\left(A^{\uparrow} \mid \eta_{Z, g} \in \Delta[T)\right)=1$ и событие $A^{\uparrow}$ под знаком вероятности в (4.6) можно убрать. Полагая $v=v_{k}=k \Delta$ и суммируя вероятности (4.6) по полуинтервалам $\Delta\left[v_{k}\right)$ при $k=0,1, \ldots$, получим

Следствие 4.1. При выполнении условий теоремы 4.1

$$
\mathbf{P}\left(\eta_{Z, g} \in \delta[T)\right)=\frac{\delta C(\alpha) P_{\alpha, g^{\prime}}}{\sqrt{T}} e^{-T D(\alpha)}(1+o(1))
$$

где

$$
P_{\alpha, g^{\prime}}=\int_{0}^{\infty} e^{-v \mu(\alpha)} P_{\alpha, g^{\prime}}(-v) d v .
$$

ДокАЗАТЕЛЬСТво ТЕОРЕМЫ 4.1. Условия теоремы 4.1 таковы, что они позволяют получить нужное утверждение путем незначительной модификации рассуждений в доказательстве теоремы 3.1. Мы предоставляем это читателю. В ряде соотношений доказательства теоремы 3.1 процесс $Z(t)$ надо заменить процессом $Z(t)+g^{\prime} t$ со сносом $g^{\prime} t$. Например, в определении события $B_{Z, T, \gamma}$ в (3.2) событие $\{\bar{Z}(T+\delta-\gamma(T+\delta)-0) \leq x\}$ следует заменить событием

$$
\left\{\sup _{t<T+\delta-\gamma(T+\delta)}\left(Z(t)+g^{\prime} t\right) \leq x\right\} .
$$

Доказательство малости условной вероятности $\mathbf{P}\left(\eta_{Z, g}<T-M \mid Z(T) \in \Delta[x+v)\right)$ при больших $M$ (cp. с (3.8), (3.10)) вытекает из условия (b) и вложения

$$
\left\{\eta_{Z, g}<T-M\right\} \in\left\{\eta_{Z^{(q)}}(x+q T)<T-M\right\} \quad \text { при } q=-r^{\prime},
$$

где $Z^{(q)}(t)=Z(t)+q t$. Дальнейшие оценки следуют рассуждениям (3.8)-(3.10), но для процесса $Z^{(q)}(t)$ и границы $x+q T, q=-r^{\prime}$.

2. ПЕРЕСЕЧЕНИЕ ГРАНИЦЫ ГОРИЗОНТАЛЬНЫМ СКАЧКОМ. Перейдем теперь к случаю $g^{\prime}<0$, когда возможно пересечение границы $g_{x}(t)$ горизонтальным скачком.

Теорема 4.2. Пусть выполнены условия теоремы 4.1, $g^{\prime}<0, \lambda_{+}>D(0)$. Тогда

$$
\begin{aligned}
& \mathbf{P}\left(\eta_{Z, g} \in \delta[T), A^{\rightarrow}, \chi(T)>v\right) \\
& \quad=\frac{\delta\left|g^{\prime}\right|}{\sqrt{T}} C(\alpha) e^{-T D(\alpha)} \int_{0}^{\infty} e^{\lambda(\alpha) u} \mathbf{P}(\tau>u+v) P_{\alpha, g^{\prime}}\left(-g^{\prime} u\right) d u(1+o(1)),
\end{aligned}
$$

где $\delta=\delta_{T}=\bar{o}(1)$ при $T \rightarrow \infty$, остаточный член равномерен по $\alpha \in K \cap[\varepsilon, \infty)$, $\varepsilon>0$, вероятность $P_{\alpha, g^{\prime}}(-v)$ определена в (4.7).

Условие $\lambda_{+}>D(0)$ введено для упрощения доказательства. Оно не является ограничительным.

ДОКАЗАТЕЛЬСТво тЕОРЕмЫ 4.2. При $g^{\prime}<0$ имеем

$$
g_{T}(T)-g_{T}(T+\delta) \sim-\delta g^{\prime}>0 \quad \text { при } \delta \rightarrow 0 .
$$


Обозначим

$$
\Delta_{g}(x]=\left(g_{T}(T+\delta), g_{T}(T)=x\right]
$$

Тогда

$$
\begin{aligned}
\mathbf{P}\left(\eta_{Z, g} \in \delta[T),\right. & \left.A^{\rightarrow}, \chi(T)>v\right) \\
& =\int_{0}^{T} \mathbf{P}\left(Z(T) \in \Delta_{g}(x], \gamma(T) \in d u, \chi(T)>v, B_{T}(u)\right)+P_{\delta[T)},
\end{aligned}
$$

где

$$
B_{T}(u)=\left\{\sup _{t \leq T-u}\left(Z(t)-g_{T}(t)\right) \leq 0\right\}, \quad P_{\delta[T)} \leq \mathbf{P}\left(Z(T+\delta) \in \Delta_{g}[x), \gamma(T+\delta)<\delta\right) .
$$

При $v>0, \delta<v$ слагаемое $P_{\delta[T)}$ в правой части (4.11) исчезает, так как событие $\{\chi(T)>\delta, \gamma(T+\delta)<\delta\}$ пусто при $\delta<v$.

Оценку первого слагаемого в правой части (4.11) разобьем на несколько этапов.

I. Аналогично предыдущему находим, что условная вероятность события $B_{T}(u)$ при условии $\left\{Z(T) \in \Delta_{g}(x], \gamma(T) \in d u, \chi(T)>v\right\}$ при каждых фиксированных $u$ и $v$ асимптотически эквивалентна при $T \rightarrow \infty$ вероятности события $P_{\alpha, g^{\prime}}\left(-g^{\prime} u\right)\left(g_{T}(T-u)-x-g^{\prime} u \sim-g^{\prime} u\right.$ при небольших $\left.u\right)$. Поэтому при фиксированном $M$

$$
\begin{aligned}
J_{0}^{M}:=\int_{0}^{M} \mathbf{P}(Z(T) & \left.\in \Delta_{g}(x], \gamma(T) \in d u, \chi(T)>v, B_{T}(u)\right) \\
& \sim \int_{0}^{M} \mathbf{P}\left(Z(T) \in \Delta_{g}(x], \gamma(T) \in d u, \chi(T)>v\right) P_{\alpha, g^{\prime}}\left(-g^{\prime} u\right) .
\end{aligned}
$$

Далее, так как функция $P_{\alpha, g^{\prime}}\left(-g^{\prime} u\right)$ возрастает по $u$, можно оценивать интеграл снизу суммами

$$
\sum_{u_{k} \leq M} \mathbf{P}\left(Z(T) \in \Delta_{g}(x], \gamma(T) \in \delta\left[u_{k}\right), \chi(T)>v\right) P_{\alpha, g^{\prime}}\left(-g^{\prime} u_{k}\right),
$$

где $u_{k}=k \delta, k=0, \ldots, N=T / \delta$ (считаем, что $N$ - целое число). В силу теоремы 1.1 и (4.10) при $u_{k}<M$ и большом фиксированном $M$

$$
\begin{aligned}
\mathbf{P}\left(Z(T) \in \Delta_{g}(x], \gamma(T) \in \delta\left[u_{k}\right), \chi(T)>v\right) & \\
= & \frac{\delta\left|g^{\prime}\right| C(\alpha)}{\sqrt{T}} e^{-T D(\alpha)}\left[I_{Z}(\alpha, u, v, \mathbb{R})-I_{Z}(\alpha, u+\delta, v, \mathbb{R})\right](1+o(1)),
\end{aligned}
$$

где

$$
I_{Z}(\alpha, u, v, \mathbb{R})-I_{Z}(\alpha, u+\delta, v, \mathbb{R}) \sim \delta e^{\lambda(\alpha) u} \mathbf{P}(\tau>u+v)
$$

при $\delta \rightarrow 0$ (пусть для простоты функция $\mathbf{P}(\tau>u)$ непрерывна).

Возвращаясь к (4.12), (4.13), находим

$$
J_{0}^{M} \geq \frac{\delta\left|g^{\prime}\right| C(\alpha)}{\sqrt{T}} e^{-T D(\alpha)} \int_{0}^{M} e^{\lambda(\alpha) u} \mathbf{P}(\tau>u+v) P_{\alpha, g^{\prime}}\left(-g^{\prime} u\right) d u(1+o(1)) .
$$


Если в (4.13) заменить $P_{\alpha, g^{\prime}}\left(-g^{\prime} u_{k}\right)$ на $P_{\alpha, g^{\prime}}\left(-g^{\prime} u_{k+1}\right)$, то получим оценку сверху для $J_{0}^{M}$, которая приводит к оценке с той же правой частью, что и в (4.14). Ясно также, что выбором $M$ интеграл в правой части (4.14) может быть сделан сколь угодно близким к интегралу от 0 до $\infty$, так что при больших $M$ правая часть в (4.14) асимптотически эквивалентна правой части в (4.9).

II. Оценим теперь сверху интеграл (см. (4.11), (4.12))

$$
\int_{M}^{T^{*}} \mathbf{P}\left(Z(T) \in \Delta_{g}(x], \gamma(T) \in d u, \chi(T)>v, B_{T}(u)\right),
$$

где $T^{*}$ таково, что значение $\alpha^{*}:=\frac{x}{T^{*}} \sim c^{*} \alpha, c^{*}>1, \alpha^{*} \in K$, по-прежнему удовлетворяет всем условиям теоремы 1.1 для $\alpha$. Этот интеграл не превосходит интеграла

$$
J_{M}^{T^{*}}:=\int_{M}^{T^{*}} \mathbf{P}\left(Z(T) \in \Delta_{g}(x], \gamma(T) \in d u, \chi(T)>v\right),
$$

который мы аналогично предыдущему будем приближать суммами

$$
\sum_{u_{k} \in\left[M, T^{*}\right]} \mathbf{P}\left(Z(T) \in \Delta_{g}(x], \gamma(T) \in \delta\left[u_{k}\right), \chi(T)>v\right),
$$

где для $k$-го слагаемого $P_{k}$ имеем

$$
\begin{aligned}
P_{k}:=\mathbf{P}\left(Z(T) \in \Delta_{g}(x], \gamma(T) \in \delta\left[u_{k}\right), \chi(T)>v\right) \\
=
\end{aligned}
$$

Обозначим

$$
A_{T, k}=\left\{Z\left(T-u_{k}\right) \in \Delta_{g}(x], \gamma\left(T-u_{k}\right)<\delta\right\} .
$$

Тогда

$$
P_{k}=\mathbf{P}\left(A_{T, k}\right) \mathbf{P}\left(\chi\left(T-u_{k}\right)>u_{k}+v \mid A_{T, k}\right),
$$

где второй множитель в правой части равен

$$
\mathbf{P}\left(\chi\left(T-u_{k}\right)>u_{k}+v \mid \gamma\left(T-u_{k}\right)<\delta\right) \leq \mathbf{P}\left(\tau>u_{k}+v\right) .
$$

Поэтому по теореме 1.1 при $\alpha_{k}=\frac{x}{T-u_{k}}$

$$
P_{k} \leq \frac{\left|g^{\prime}\right| \delta^{2} C\left(\alpha_{k}\right)}{\sqrt{T-u_{k}}} e^{-\left(T-u_{k}\right) D\left(\alpha_{k}\right)} \mathbf{P}\left(\tau>u_{k}+v\right)(1+o(1)) .
$$

Здесь

$$
\left(T-u_{k}\right) D\left(\alpha_{k}\right)=T \mathbf{D}\left(1-\rho_{k}, \alpha\right) \quad \text { при } \rho_{k}=\frac{u_{k}}{T},
$$

где $\mathbf{D}(t, \alpha)=\sup _{(\lambda, \mu) \in \partial \mathscr{A}<0}(\lambda t+\mu \alpha), \mathbf{D}(1, \alpha)=D(\alpha)$ (см. [1]). В силу выпуклости функции $\mathbf{D}(t, \alpha)$ и равенства $\frac{\partial}{\partial t} \mathbf{D}(t, \alpha)=\lambda(\alpha / t)$ (см. [1])

$$
\mathbf{D}\left(1-\rho_{k}, \alpha\right) \geq D(\alpha)-\rho_{k} \lambda(\alpha)
$$

и, стало быть, при всех $k$

$$
\left(T-u_{k}\right) D\left(\alpha_{k}\right) \geq T D(\alpha)-u_{k} \lambda(\alpha),
$$




$$
\begin{gathered}
\sum_{u_{k} \in\left[M, T^{*}\right]} P_{k}<\frac{\left|g^{\prime}\right| \delta e^{-T D(\alpha)}}{\sqrt{T-T^{*}}} \sum_{u_{k} \in\left[M, T^{*}\right]} \delta C\left(\alpha_{k}\right) e^{u_{k} \lambda(\alpha)} \mathbf{P}\left(\tau>u_{k}+v\right)(1+o(1)) \\
=\frac{c\left|g^{\prime}\right| \delta e^{-T D(\alpha)}}{\sqrt{T-T^{*}}} \int_{M}^{\infty} e^{u \lambda(\alpha)} \mathbf{P}(\tau>u+v) d u(1+o(1))
\end{gathered}
$$

при некотором $c$. Так как $\lambda_{+}>D(0)$, то $\lambda(\alpha)<\lambda_{+}$и интеграл в (4.20) сходится к 0 при $M \rightarrow \infty$. Отсюда следует, что

$$
J_{M}^{T^{*}}=o\left(J_{0}^{M}\right) \quad \text { при } M \rightarrow \infty, T \rightarrow \infty .
$$

III. Оценим далее

$$
J_{T^{*}}^{(1-\varepsilon) T}=\int_{T^{*}}^{(1-\varepsilon) T} \mathbf{P}\left(Z(T) \in \Delta_{g}(x], \gamma(T) \in d u, \chi(T)>v\right)
$$

при малом фиксированном $\varepsilon>0$. Как и в п. II доказательства, воспользуемся приближением интеграла суммами и неравенством (4.18), в силу которого (см. (4.18), (4.19))

$$
P_{k} \leq \mathbf{P}\left(A_{T, k}\right) \mathbf{P}\left(\tau>u_{k}+v\right) .
$$

Для получения требуемой оценки сверху для

$$
\boldsymbol{\Sigma}_{*}:=\sum_{u_{k} \in\left[(1-\varepsilon) T, T^{*}\right]} P_{k}
$$

воспользуемся более грубыми, чем в п. ІІ, подходами. В силу ПБУ для ОПВ в случае $\lambda_{+}>D(0)$ при $T \rightarrow \infty$ имеем

$$
\ln \mathbf{P}\left(A_{T, k}\right) \leq \ln \mathbf{P}\left(Z\left(T-u_{k}\right) \in \Delta_{g}(x]\right) \leq-\left(T-u_{k}\right) D\left(\frac{x}{T-u_{k}}\right)(1+o(1))
$$

и в силу (4.19) аналогично предыдущему

$$
\begin{gathered}
\ln \mathbf{P}\left(A_{T, k}\right) \leq-T\left(1-\rho_{k}\right) D\left(\frac{\alpha}{1-\rho_{k}}\right)=-T \mathbf{D}\left(1-\rho_{k}, \alpha\right) \leq-T D(\alpha)+u_{k} \lambda(\alpha), \\
\boldsymbol{\Sigma}_{*} \leq \frac{c}{\delta} e^{-T D(\alpha)} \int_{T^{*}}^{(1-\varepsilon) T} e^{u \lambda(\alpha)} \mathbf{P}(\tau>u+v) d u
\end{gathered}
$$

при некотором $c<\infty$. Так как $\lambda(\alpha)<\lambda_{+}-\varepsilon$ при некотором $\varepsilon>0$, то

$$
\boldsymbol{\Sigma}_{*} \leq \frac{c e^{-T D(\alpha)}}{\delta} e^{-\varepsilon_{1} T}
$$

при некотором $\varepsilon_{1}>0$. Таким образом, значение интеграла $J_{T^{*}}^{(1-\varepsilon) T}$ пренебрежимо мало по сравнению с $J_{0}^{M}$.

IV. Оценим

$$
\begin{aligned}
J_{(1-\varepsilon) T}^{T}=\int_{(1-\varepsilon) T}^{T} \mathbf{P}\left(Z(T) \in \Delta_{g}(x], \gamma(T)\right. & \in d u) \\
& \leq \mathbf{P}(\bar{Z}(\varepsilon T)>x) \mathbf{P}(\tau>(1-\varepsilon) T) .
\end{aligned}
$$


В силу теоремы 3.1 в [3] при всех $\alpha>0$ и достаточно малом $\varepsilon$ выполняется

$$
\mathbf{P}(\bar{Z}(\varepsilon T)>x) \leq \exp \left\{-\varepsilon T D\left(\frac{x}{\varepsilon T}\right)+o(T)\right\}
$$

при $T \rightarrow \infty$, где в силу (4.19)

$$
\varepsilon T D\left(\frac{\alpha}{\varepsilon}\right)=T \mathbf{D}(\varepsilon, \alpha) \geq T D(\alpha)-T \lambda(\alpha)(1-\varepsilon) .
$$

Вместе с (4.21) это дает

$$
J_{(1-\varepsilon) T}^{T} \leq e^{-T D(\alpha)+\lambda(\alpha)(1-\varepsilon) T+o(T)} \mathbf{P}(\tau>(1-\varepsilon) T) \leq e^{-T D(\alpha)-\varepsilon_{1} T}
$$

при некотором $\varepsilon_{1}>0$. Это вновь доказывает пренебрежимую малость значения рассматриваемого интеграла по сравнению с $J_{0}^{M}$.

V. Остается оценить (см. (4.11))

$$
P_{\delta[T)} \leq \mathbf{P}\left(Z(T+\delta) \in \Delta_{g}(x], \gamma(T+\delta)<\delta\right) .
$$

В силу теоремы 1.1

$$
P_{\delta[T)} \leq \frac{c \delta^{2}}{\sqrt{T}} e^{-T D(\alpha)}=o\left(J_{0}^{M}\right)
$$

при $\delta \rightarrow 0$ и некотором $c<\infty$.

Полученные оценки доказывают (4.9). Теорема 4.2 доказана.

4.2. Интегро-локальные теоремы в задаче о вероятности разорения в теории страхования. Задача о вероятности разорения страховой компании состоит в следующем. Пусть $T_{1}, T_{2}, \ldots$ - моменты наступления крупных выплат по страховым случаям, а $\zeta_{1}, \zeta_{2}, \ldots$ - размеры этих выплат соответственно. Пусть далее $r$ - средняя «скорость» поступлений страховых взносов (суммы, поступающие в компанию от страхователей за единицу времени). Тогда если $x$ - начальный капитал компании, то ее капитал в момент $t$ будет примерно равен $x+r t-Z(t)$. Это значит, что если

$$
\inf _{t \leq T}(x+r t-Z(t))<0,
$$

то компания к моменту $T$ разорится. Задача состоит в отыскании приближений для вероятности разорения и распределения времени разорения (если таковое произойдет). Это есть классическая задача о разорении. Ей посвящено значительное количество работ, включая монографии (см., например, [5-8]).

Введем в рассмотрение ОПВ $Z^{(q)}(t)$ с линейным сносом $q t$ :

$$
Z^{(q)}(t)=Z(t)+q t
$$

и «обычный» ОПВ (без сноса) $Z^{[q]}(t)$, управляемый скачками $\left(\tau, \zeta^{(q)}\right)$ :

$$
\zeta^{(q)}=\zeta+q \tau,
$$

так что $Z^{(q)}\left(T_{k}\right)=Z^{[q]}\left(T_{k}\right)$. Ясно, что если $q \leq 0$, то

$$
\bar{Z}^{(q)}(T):=\max _{t \leq T} Z^{(q)}(t)=\bar{Z}^{[q]}(T)
$$

и $\eta_{Z^{(q)}}(x)=\eta_{Z^{[q]}}(x)$ есть момент разорения компании (несобственная случайная величина). Таким образом, задача о пересечении высокого уровня $x$ процессом 
$Z^{(q)}$ со сносом $q t$ при $q \leq 0$ сводится к той же задаче для «обычного» ОПВ $Z^{[q]}$ со скачками $\left(\tau, \zeta^{(q)}\right)$.

Далее, событие (4.22) можно при $q=-r<0$ записать в виде

$$
\left\{\bar{Z}^{(q)}(T)>x\right\}=\left\{\bar{Z}^{[q]}(T)>x\right\}
$$

и, кроме того,

$$
\left\{\eta_{Z^{(q)}}(x)<T, \chi_{Z^{(q)}}(x)<v\right\}=\left\{\eta_{Z^{[q]}}(x)<T, \chi_{Z^{[q]}}(x)<v\right\} .
$$

Для отыскания вероятностей этих событий понадобятся интегро-локальные вероятности

$$
\mathbf{P}\left(\eta_{Z^{[q]}}(x) \in \delta[T), \chi_{Z^{[q]}}(x)<v\right) .
$$

Они найдены в следствии 3.1. Чтобы воспользоваться этим следствием, надо «пересчитать» множители $C(\alpha), J_{\alpha}(v)$ и функцию $D(\alpha)$ для вектора $\left(\tau, \zeta^{(q)}\right)$. Для этого достаточно найти базовую функцию $A^{(q)}(\mu)$, соответствующую управляющему вектору $\left(\tau, \zeta^{(q)}\right)$. Имеем

$$
\mathbf{A}^{(q)}(\lambda, \mu):=\ln \mathbf{E} e^{\lambda \tau+\mu \zeta^{(q)}}=\ln \mathbf{E} e^{\lambda \tau+\mu(\zeta+q \tau)}=\mathbf{A}(\lambda+q \mu, \mu) .
$$

Отсюда и из определения базовой функции находим

$$
A^{(q)}(\mu)=q \mu+A(\mu)
$$

(например, в области аналитичности $\mathbf{A}^{(q)}\left(-A^{(q)}(\mu), \mu\right)=\mathbf{A}(-q \mu-A(\mu)+q \mu, \mu)=$ $0)$. Применяя к $A^{(q)}(\mu)$ преобразование Лежандра, получаем $D^{(q)}(\alpha)=D(\alpha-q)$. Отметим, что последнее соотношение, а вместе с ним и (4.24) можно получить также из принципа больших уклонений для процесса $Z^{(q)}(t)$ (см. [2]; мы используем обозначение $Z^{(q)}$, так как оно использовалось в [2]).

Таким образом, искомую асимптотику вероятностей (4.23) можно получить с помощью леммы 3.1, но при этом приходится «пересчитывать» названные выше функции в правой части (3.12).

Другая возможность найти асимптотику (4.23) состоит в использовании результатов $\S 4$. При этом «пересчитывать» придется не функции, а лишь параметр $\alpha$. Действительно, вероятность разорения за время $T$, равная

$$
\mathbf{P}\left(\sup _{t \leq T}(Z(t)-r t)>x\right),
$$

есть вероятность пересечения ОПВ $Z(t)$ границы

$$
g_{T}(t)=x+r t=x^{(r)}+r(t-T) \quad \text { при } x^{(r)}=x+r T .
$$

Ясно, что такая граница удовлетворяет условиям (a), (b) (см. (4.3), (4.4)) при $g^{\prime}=r$. В этих условиях вместо $x$ и $\alpha$ следует использовать параметры $x^{(r)}$ и $\alpha^{(r)}=x^{(r)} / T=\alpha+r>r$ соответственно. Такие параметры исключают возможность пересечения границы горизонтальным скачком. При этом

$$
\eta_{Z^{(q)}}(x)=\eta_{Z, g} \quad \text { при } q=-r .
$$

Чтобы получить содержательное утверждение, мы будем предполагать, что значения $\alpha=x / T$ и $\alpha^{(r)}-a$ отделены от 0 .

Из теоремы 4.1 вытекает 
Следствие 4.2. Пусть $\boldsymbol{\xi}$ - нерешетчатый вектор, $r>a, \alpha=x / T>\varepsilon$ при некотором $\varepsilon>0$. Тогда

$$
\mathbf{P}\left(\eta_{Z, g} \in \delta[T), \chi_{Z, g} \in \Delta[v)\right)=\frac{\Delta \delta C\left(\alpha^{(r)}\right)}{\sqrt{T}} e^{-T D\left(\alpha^{(r)}\right)-v \mu\left(\alpha^{(r)}\right)} P_{\alpha^{(r)}, r}(-v)(1+o(1)),
$$

где $\Delta=\Delta_{T}=\bar{o}(1), \delta=\delta_{T}=\bar{o}(1)$ при $T \rightarrow \infty$, остаточный член о(1) равномерен по $\alpha \in K \cap\left\{\alpha^{(r)} \geq \varepsilon\right\}$.

Из следствия 4.1 вытекает

Следствие 4.3. При выполнении условий следствия 4.2

$$
\mathbf{P}\left(\eta_{Z, g} \in \delta[T)\right)=\frac{\delta C\left(\alpha^{(r)}\right) P_{\alpha^{(r)}, r}}{\sqrt{T}} e^{-T D\left(\alpha^{(r)}\right)}(1+o(1)),
$$

где множитель $P_{\alpha, r}$ определен в (4.8).

Отыскание интегральных вероятностей $\mathbf{P}\left(\eta_{Z, g}>T\right)$ с помощью полученных утверждений будет произведено в другой работе.

\section{ЛИТЕРАТУРА}

1. Боровков А. А., Могульский А. А. Интегро-локальные предельные теоремы для обобщенных процессов восстановления при выполнении условия Крамера. I, II // Сиб. мат. журн. 2018. Т. 59, № 3. С. 491-514; № 4. С. 731-750.

2. Боровков А. А., Могульский А. А. Принципы больших уклонений для конечномерных распределений обобщенных процессов восстановления // Сиб. мат. журн. 2015. Т. 56, № 1. C. $36-64$.

3. Боровков А. А. Асимптотический анализ случайных блужданий. Быстроубывающие распределения скачков. М.: Физматлит, 2013.

4. Боровков А. А. Принципы больших уклонений в граничных задачах для обобщенных процессов восстановления // Сиб. мат. журн. 2016. Т. 57, № 3. С. 562-595.

5. Asmussen S., Albrecher H. Ruin probabilities. Singapore: World Sci., 2010.

6. von Bahr B. Ruin probabilities expressed in terms of ladder height distributions // Scand. Actuar. J. 1974. N 4. P. 190-204.

7. Drekic S., Willmot G. E. On the density and moments of the time of ruin with exponential claims // Astin Bull. 2003. V. 33, N 1. P. 11-21.

8. Asmussen $S$. Approximations for the probability of ruin within finite time // Scand. Actuar. J. 1984. P. 31-57.

Поступила в редакцию 27 августа 2019 г.

После доработки 26 сентлбря 2019 г.

Принята к публикачии 18 октлбря 2019 г.

Боровков Александр Алексеевич Институт математики им. С. Л. Соболева СО РАН, пр. Академика Коптюга, 4, Новосибирск 630090

borovkov@math.nsc.ru 